This is an author produced version of a paper published in Neurobiology of Disease. This paper has been peer-reviewed but does not include the final publisher proof-corrections or journal pagination.

Citation for the published paper:

Carlsson, Thomas and Winkler, Christian and Lundblad, Martin and Cenci, M Angela and Bjorklund, Anders and Kirik, Deniz "Graft placement and uneven pattern of reinnervation in the striatum is important for development of graft-induced dyskinesia." Neurobiol Dis. 2005 Oct 24; [Epub ahead of print] http://dx.doi.org/10.1016/j.nbd.2005.09.008

Access to the published version may require journal subscription. Published with permission from: Elsevier 


\section{Graft placement and uneven pattern of reinnervation in the striatum is important for development of graft-induced dyskinesia}

Thomas Carlsson $^{1}$, Christian Winkler ${ }^{1,2}$, Martin Lundblad ${ }^{1}$, M. Angela Cenci ${ }^{1}$, Anders Björklund $^{1}$ and Deniz Kirik ${ }^{1}$

1. Wallenberg Neuroscience Center, Department of Experimental Medical Science, Lund University, BMC A11, 221 84, Lund, Sweden

2. Department of Neurology, Hannover Medical School, 30625 Hannover, Germany

Correspondence should be addressed to: Thomas Carlsson

Wallenberg Neuroscience Center

BMC A11, 221 84, Lund, Sweden

Tel: +46-46-2220527,

Fax: +46-46-2223436

E-mail: Thomas.Carlsson@med.lu.se

Keywords: Parkinson's disease; dyskinesia; L-DOPA; cell transplantation; ventral mesenchephalon; motor behavior. 


\section{Abstract}

In two recent double-blind clinical trials of fetal ventral mesencephalic cell transplants into the striatum in patients with Parkinson's disease (PD), a significant proportion of the grafted patients developed dyskinetic side effects, which were not seen in the sham operated patients. Comparison between dyskinetic and non-dyskinetic grafted patients in one of the trials suggested that an uneven pattern of striatal reinnervation might be the leading cause of the dyskinesias. Here we studied the importance of graft placement for the development of dyskinesias in parkinsonian rats. Abnormal involuntary movements resembling peak-dose dyskinesias seen in PD patients were induced by daily injections of L-DOPA for six weeks. The dyskinetic animals received about 130.000 fetal ventral mesencephalic cells as single grafts placement in the rostral or the caudal aspect of the head of striatum. The results show that grafts placed in the caudal, but not the rostral, part are effective in reducing the L-DOPA-induced limb and orolingual dyskinesia, predominantly seen as hyperkinesia. The same grafts, however, also induced a new type of dyskinetic behavior after activation with amphetamine, which were not seen in nongrafted lesion controls. The severity of these abnormal involuntary movements was significantly correlated with a higher graft-derived dopaminergic reinnervation in the caudal aspect of the head of striatum relative to the rostral part. The results indicate that graft-induced dyskinesias in PD patients may be linked to single, small graft deposits that provide an uneven, patchy reinnervation of the putamen. 


\section{Introduction}

Current pharmacological treatment of Parkinson's disease (PD) is primarily based on oral administration of L-3,4-dihydroxyphenylalanine (L-DOPA), the precursor of dopamine (DA). However, high incidence of complications including, among others, induction of abnormal involuntary movements or postures, termed dyskinesias, is a major concern (Marsden et al., 1982, Quinn, 1998, Fahn, 2000). Observations in PD patients, as well as data obtained from animal experiments, suggest that fluctuations in striatal DA concentration as a result of the pulsatile L-DOPA administration is one of the main contributors to the development of these side effects (Fahn, 2000, Jenner, 2000, Nutt et al., 2000, Carlsson et al., 2005).

Transplantation of fetal DA neurons is currently being explored as a restorative treatment strategy for PD patients. As grafted DA neurons provide a new fiber terminal network in the striatum, patients suffering from L-DOPA-induced dyskinesias would be expected to improve after transplantation. In open-label clinical transplantation studies, reductions in dyskinesias (\% time in "on" with dyskinesias) have been reported in some patients, while the remaining patients either did not benefit or had worsened (Defer et al., 1996, Hagell et al., 1999, Hauser et al., 1999, Brundin et al., 2000). The reason for this variable response remains unclear. Two recent doubleblind placebo-controlled trials (Freed et al., 2001, Olanow et al., 2003) investigating the usefulness of fetal DA neuron transplantation in PD patients have raised concerns that the grafts may induce a new type of dyskinesia (Hagell et al., 2002, Lindvall and Bjorklund, 2004, Winkler et al., 2005). In the first study, 4 out of 33 grafted patients developed severe involuntary movements (Greene et al., 1999, Freed et al., 2001), and in the second study, off-state dyskinesia 
was observed in 13 out of 23 grafted patients (Olanow et al., 2003). These graft-induced dyskinesias (GIDs), which persisted after L-DOPA withdrawal, were generally mild, but were disabling and required surgery in 3 cases (Freed et al., 2004). Further investigation of the patients from the first trial, using $\left[{ }^{18} \mathrm{~F}\right]$-DOPA positron emission tomography (PET), showed that in the dyskinetic patients there were asymmetric and localized increases in the PET signal in the ventral putamen, while this was not seen in the grafted non-dyskinetic patients (Ma et al., 2002). This lead to the conclusion that focal, unbalanced increases in striatal DA function caused by a patchy DA innervation may contribute to the development of these GIDs.

In the present study, we have investigated the effect of DA neuron grafts, and the role of graft placement, on L-DOPA-induced dyskinesia in the 6-hydroxydopamine (6-OHDA) lesioned rats, and explored to what extent an uneven, patchy pattern of reinnervation could result in development of GIDs. We studied the effect of single grafts deposits implanted in already dyskinetic animals, i.e. animals that had developed dyskinesia as a result of chronic intermittent L-DOPA treatment prior to grafting. In the dyskinesia model used here (Cenci et al., 1998, Lee et al., 2000, Lundblad et al., 2002, Winkler et al., 2002) stable and long-lasting dyskinesia involving forelimb, orolingual and axial regions, are induced over a 3-4-week period of daily LDOPA injections in 6-OHDA lesioned rats. In this experiment we selected two graft sites; one in the caudal aspect of the head of striatum, close to the area that is known to show long-lasting upregulation of cellular activity markers in this model; and one in the rostral aspect of the head of striatum, further away from this area. 


\section{Materials and methods:}

\section{Experimental design}

A total of 125 adult female Sprague-Dawley rats weighing 225-250 grams were used in the present study (B\&K Universal, Stockholm, Sweden). The animals were housed under a $12 \mathrm{hr}$ light / $12 \mathrm{hr}$ dark cycle with free access to water and food. All surgical procedures were performed according to the regulations set by the Ethical Committee for use of Laboratory animals at Lund University.

The animals received a unilateral 6-OHDA lesion placed at the medial forebrain bundle (MFB) (Fig. 1). Lesioned rats were screened behaviorally using the amphetamine-induced rotation test. Eighty-four rats exhibited full body rotations of $\geq 6$ turns/min towards the side of dopamine deficiency. Seventy-four rats were then treated with daily injections of L-DOPA at a dose of 6 $\mathrm{mg} / \mathrm{kg}$ for six weeks until the abnormal involuntary movements (AIMs; equivalent to peak-dose dyskinesia seen in PD patients) were fully developed and stabilized. Out of 49 moderate to severely dyskinetic rats 21 were allocated to three groups balanced on basis of their dyskinesia scores. Two groups of dyskinetic animals received transplantation of fetal ventral mesencephalic (VM) tissue in the rostral aspect of the head of striatum $(n=7)$ or in the caudal part $(n=7)$. A third group of dyskinetic rats [Les-Ctrl (L-DOPA), n=7] was sham-operated, and did not receive any graft. In addition, we included a group of lesioned but L-DOPA-naive rats as controls [Les-Ctrl (Drug-Naive), $\mathrm{n}=8$ ]. Post-graft testing was initiated 1 week after transplantation surgery. The dyskinetic animals continued to receive L-DOPA injections at the maintenance dose (see below), while the drug-naive lesion control group was injected with vehicle only. During the follow-up period L-DOPA-induced AIMs were repeatedly tested up to 24 weeks. At 13 weeks a second 
amphetamine-induced rotation test was conducted to assess the functionality of the grafts. In addition, at 20 and 30 weeks after grafting we injected the animals with amphetamine in order to stimulate DA release from the graft. The animals were then analyzed for appearance of abnormal movements. After completion of the behavioral tests at 30 weeks post grafting, the animals were killed and the brains perfused for further histological analysis.

\section{6-OHDA lesion}

All surgical procedures were performed under anesthesia induced by an injectable 1:1 mixture of Hypnorm and Dormicum (Apoteksbolaget, Sweden). The surgery was made using a stereotaxic frame (Stoelting, Wood Dale, Illinois) with a $5 \mu$ l Hamilton syringe attached. In order to achieve a complete lesion of the nigrostriatal DA system all animals received two injections of $7 \mu \mathrm{g}$ and $8.75 \mu \mathrm{g}$ of 6-OHDA (calculated as free base) in the MFB at a concentration of $3.5 \mu \mathrm{g} / \mu \mathrm{l}$ in 0.2 $\mathrm{mg} / \mathrm{ml}$ ascorbate in saline (Sigma-Aldrich $\mathrm{AB}$, Sweden). The coordinates were calculated using bregma as a reference with respect to the anteroposterior (AP) and the mediolateral (ML) coordinates, using the rat brain atlas (Paxinos and Watson, 1998) and were as follows: (1) AP: 4.4 ML: -1.2 ; (2) AP: -4.0, ML: -0.8. The dorsoventral (DV) positions of the injections were -7.8 and $-8.0 \mathrm{~mm}$ below the dura, and the tooth bar was set to -2.4 and $+3.4 \mathrm{~mm}$ for the two lesion sites, respectively. The 6-OHDA was injected at a rate of $1.0 \mu 1 / \mathrm{min}$ and the syringe was kept in place for an additional 3 min before it was slowly retracted.

\section{Transplantation procedure}

The VM tissue was prepared into a single cell suspension following the procedure described earlier (Nikkhah et al., 1994, Winkler et al., 1999, Kirik et al., 2001, Georgievska et al., 2004). Briefly, VM tissue from E13.5 rat embryos was dissected out and incubated in $0.1 \%$ trypsin, 
0.05\% DNase in Dulbecco's modified eagle medium (DMEM) for $30 \mathrm{~min}$ at $37^{\circ} \mathrm{C}$. The tissue was mechanically dissociated into a single-cell suspension and concentrated by centrifugation at $500 \mathrm{rpm}$. The pellet was re-suspended in DMEM into about 130.000 cells $/ \mu \mathrm{l}$ with $>99 \%$ viability.

All transplantation surgery was performed using a $5 \mu \mathrm{l}$ Hamilton syringe fitted with a glass capillary (outer diameter of $60-80 \mu \mathrm{m})$. The Rostral graft group $(\mathrm{n}=7)$ received injection of 130.000 cells in the rostral aspect of the head of striatum (at the coordinate AP: $+1.2 \mathrm{~mm}$, ML: $2.7 \mathrm{~mm})$ while the Caudal graft group $(\mathrm{n}=7)$ received injection of same number of cells in the caudal aspect of the head of striatum (at the coordinate AP: $+0.2 \mathrm{~mm}$ and ML: $-3.5 \mathrm{~mm}$ ). In both cases the tooth bar was set at 0.0 and at each site, the injection was made as two $0.5 \mu$ deposits at the DV coordinates $-5.0 \mathrm{~mm}$ and $-4.0 \mathrm{~mm}$ below the dura. After the injection was completed the needle was kept in place for an additional $2 \mathrm{~min}$ before it was slowly retracted. After the transplantation the survival of cells in the remaining suspension was determined at about 110.000 cells/ $\mu$ with a viability of about $95 \%$.

\section{Behavioral analysis}

Amphetamine-induced rotation was monitored, following an i.p. injection of $2.5 \mathrm{mg} / \mathrm{kg}$ of damphetamine sulfate (Apoteksbolaget, Sweden), at 2 weeks after the 6-OHDA lesion and 13 weeks after the grafting. Right and left body turns were recorded over 90 min using automated rotometer bowls (AccuScan Instrument Inc., Columbus, Ohio). The data are expressed as net full body turns per min, where rotation towards the side of the lesion was given a positive value.

L-DOPA-induced dyskinesia: A dose of $6 \mathrm{mg} / \mathrm{kg}$ L-DOPA methyl ester (Research Organics, Cleveland, Ohio) combined with the peripheral DOPA-decarboxylase inhibitor, benserazide- 
$\mathrm{HCl}$, (15 mg/kg, Sigma-Aldrich, Sweden), was dissolved in physiological saline and administered daily to each rat as an i.p. injection in order to induce dyskinesia. Stable AIM scores were obtained by daily L-DOPA injections in the majority of the animal over six weeks. After the plateau was reached animals were selected for high AIM scores and kept on a maintenance regimen of twice weekly L-DOPA injections, as described by Lee et al., (2000), at the same dose for 29 weeks. The severity of AIMs were determined six times during the induction period before grafting, and at 2, 6, 12, 18 and 24 weeks after the transplantation. All tests were evaluated according to the rat dyskinesia scale described in detail previously (Lee et al., 2000, Lundblad et al., 2002), with some minor modifications (Winkler et al., 2002, Carlsson et al., 2005). Each time-point represents the average of two consecutive test sessions performed 3-4 days apart. Briefly, the animals were placed individually in transparent plastic cages without bedding material and scored every 30 min for a 4-hour period following the injection of LDOPA. We classified AIMs into four subtypes according to their topographic distribution as forelimb (Li), orolingual (Ol) axial (Ax), and locomotive (Lo) behaviors (see movie 1). The forelimb and orolingual dyskinesia are predominantly hyperkinesia, while the axial dyskinesia is essentially of a dystonic type. The locomotive dyskinesia was expressed as circling movements away from the lesioned side. Enhanced manifestations of normal behaviors such as grooming, gnawing, rearing and sniffing were not included in the rating. The severity of the AIMs was assessed using the scores 0 to 4 for each of the four AIM subtypes (0: Absent; 1: Occasional i.e. present during less than $50 \%$ of the observation time; 1.5: Present $50 \%$ of the observation time; 2: Frequent, i.e. present during more than $50 \%$ of the observation time; 2.5: Present most of the time with very short duration of non-dyskinetic episodes; 3: Continuous, but interrupted by repeated strong sensory stimuli, e.g. sudden noise, opening of the cage lid; 3.5: Continuous, but 
inconsistently interrupted by repeated strong sensory stimuli; 4: Continuous, not interrupted by repeated strong sensory stimuli). Integrated AIM scores were calculated for each animal as the area under the curve in the raw data plot of AIM scores over the whole observation time of 4 hours.

Graft-induced dyskinesia test: In order to investigate and quantify the GIDs (see movie 2), AIM tests were conducted at the 20 and 30 weeks after grafting following injection of $2.5 \mathrm{mg} / \mathrm{kg} \mathrm{d}$ amphetamine sulfate (Apoteksbolaget, Sweden). The two tests were performed at least 48 hour after the last L-DOPA injections to minimize the interference between the drugs. These AIM tests were evaluated at 20 and 30 weeks using the same rating scale as for analysis of L-DOPAinduced dyskinesia. AIMs were monitored every $10 \mathrm{~min}$ for a period of 3 hours. In addition, to these abnormal behaviors a different type, not seen after L-DOPA injections, was observed in the grafted animals under the effect of amphetamine. This behavior consists in wide circular and vertical asymmetric movements of the head, towards the side contralateral to the lesion and grafted side. We referred to these as asymmetric head-nodding behaviors (see movie 3). GIDs were also investigated prior to the 18-week time-point in absence of pharmacological stimulation, i.e. under baseline conditions and after the application of stressful stimuli by injection of saline and tail pinch (plastic clip attached to the tail of the rat). AIMs were evaluated at 15, 30 and $45 \mathrm{~min}$ after saline injection and at 5 and $10 \mathrm{~min}$ after the application of the plastic clip to the tail.

Inclusion criteria in the study. Following the 6-OHDA lesion and the first amphetamine rotation animals with that exhibited at least 6 turns/min were included for L-DOPA challenge. At the end of the daily L-DOPA injection period animals with an integrated Li+Ol+Ax AIM score of $>470$ points per session were considered moderate to severely dyskinetic and were allocated into 
experimental groups to receive either fetal tissue grafts or sham surgery. A second rotation test was conducted at 13 weeks post transplantation to evaluate the presence of a functional graft, which was used as criteria to complete the experiment. Two animals in Rostral graft group and one animal in Caudal graft group were excluded from the study, as they showed no evidence of a surviving graft at this stage.

\section{Histological analysis}

At the end of the experiment (30 weeks post grafting), the animals were killed 3 hours following the administration of $2.5 \mathrm{mg} / \mathrm{kg} \mathrm{d}$-amphetamine sulfate. After being deeply anesthetized with sodium pentobarbital (Apoteksbolaget, Sweden), the animals were transcardially perfused with $50 \mathrm{ml}$ physiological saline followed by $250 \mathrm{ml}$ ice-cold paraformaldehyde in $0.1 \mathrm{M}$ phosphate buffer ( $\mathrm{pH}$ 7.4) over $5 \mathrm{~min}$. The brains were then removed and postfixed for additional 2 hours in the same fixative and thereafter transferred into $25 \%$ sucrose, and cut at $40 \mu \mathrm{m}$ thickness in 8 series using a freezing slide-microtome (SM2000R, Leica).

Immunohistochemistry was conducted to visualize the tyrosine hydroxylase (TH)-positive cell bodies and their fiber terminals. Free-floating sections were quenched for 10 min in $3 \% \mathrm{H}_{2} \mathrm{O}_{2}$ and $10 \%$ methanol in potassium-phosphate buffer (KPBS). This was followed by a preincubation step for one hour in 5\% normal horse serum, $0.25 \%$ triton-X in KPBS and later over-night incubation with the primary antibody against $\mathrm{TH}$ (MAB 318, mouse $\operatorname{IgG}, 1: 4,000$, Chemicon, $\mathrm{CA}$ ) in room temperature. On the second day, one-hour incubation in a biotinylated secondary antibody (BA 2001, horse anti-mouse, 1:200, Vector Laboratories, Burlingame, CA) was followed by one-hour incubation in avidin-biotin-peroxidase solution (ABC Elite, vector Laboratories). The reaction was visualized using the chromogen 3, 3'-diaminobenzidine and 
$0.01 \% \mathrm{H}_{2} \mathrm{O}_{2}$. The sections were then mounted on chrom-alum-coated glass slides and coverslipped with Depex after dehydration in alcohol and clearing in xylene.

Stereological estimation of $\mathbf{T H}$-positive cell numbers was performed using 40x 1.30 oil objective with the Olympus CAST system version 2.0 (Olympus, Denmark A/S, Albertslund, Denmark). The grafts position in the striatum were delineated and a counting frame was randomly placed on the first counting area and moved systematically throughout the inclusion area (Kirik et al., 2001). Guard volumes of $3 \mu \mathrm{m}$ from the top and the bottom of the section were excluded to avoid problems with lost caps. The antibody penetration was determined by registration of the depth of each counted neuronal profile that appeared in focus within the counting frame. The analysis of the cells' position revealed an incomplete penetration of antibody leaving 4-5 $\mu \mathrm{m}$ in the center unstained. The inclusion volume to estimate total number of cells was therefore calculated excluding this portion of the sections, and was done according to the optical fractionator principle as described by West (1999).

Striatal TH-positive fiber density measurements were assessed by measuring the mean optical density using a digital camera (ProgRes C14, Jena, Germany) and the Image J software version 1.32 for Mac OS X platform (National Institutes of Health, USA, http://reb.info.nih.gov/ij/). The analyses were performed on four selected sections at the following coordinates: $+1.60,+1.20$, +0.20 and 0.0 using bregma as the reference. The two anterior levels constituted values from rostral striatum, whereas the latter two levels were reported as caudal striatum. The inclusion area for striatum was drawn from the lateral ventricle, the external capsule and a horizontal line connecting the ventral end of the ventricle via the anterior commissure to the external capsule. On the sections where graft cores were identified, the area including the cell bodies was excluded 
from the delineation. The data are expressed as percent of intact side and represents the average of either the two levels at the rostral or the caudal striatum.

\section{Statistical analysis}

Group comparisons were performed using either one-way factorial ANOVA or two-way repeated measures ANOVA where appropriate. Posthoc analysis was performed using the Student t-test or the Tukey HSD analysis. Linear regression analyses were performed between dyskinesias (L-DOPA- or graft-induced) and the quantitative morphological data where appropriate regression coefficients and significances are reported in the respective figures. Statistical significance was set at $\mathrm{P}<0.05$, for all analyses. All statistics in this study were performed using the JMP Statistical software version 5.0.1.2 (SAS Institute Inc., Cary, NC, USA). 


\section{Results}

The aim of the present study was, first, to investigate the effect of graft placement on modulation of L-DOPA-induced dyskinesia in a rodent model of PD, and secondly, to investigate whether fetal DA neuron grafts can induce dyskinesia ("GID”) in rats. For this purpose, we generated rats with near complete lesion of the ascending dopaminergic pathway by 6-OHDA injections in the MFB, and characterized the motor behavioral impairment using amphetamine-induced rotation (Fig. 1). The animals were then treated with daily i.p. injections of L-DOPA for 6 week, during which time they gradually developed and further stabilized a dyskinetic response. The rats displaying moderate to severe dyskinesias were allocated to one of two transplantation groups (Rostral graft or Caudal graft) or a sham group [Les-Ctrl (L-DOPA)]. The two transplanted groups received single grafts into the rostral (Fig. 2E, F), or the caudal aspect of the head of striatum (Fig. 2G, H), respectively. A further control group was neither L-DOPA treated nor grafted [Les-Ctrl (Drug-Naive)]. All animals were followed by repeated behavioral testing prior to killing at 30 weeks post-grafting.

\section{Graft survival and fiber outgrowth into the host striatum}

TH-immunohistochemistry revealed surviving DA neuron grafts in all transplanted animals included in the study (Fig 2E-H). The number of surviving TH-positive cells were determined by stereological estimation tools and the fractionator principle. In the Les-Ctrl groups, no THpositive cell bodies were observed in any of the striatal sections on the lesioned side (Fig. 2C, D). The average cell numbers were estimated at $3339 \pm 163$ cells in the Rostral graft group and $2577 \pm 247$ in the Caudal graft group (Fig. 3A). 
Striatal TH-positive fiber density was evaluated in two sections at the rostral graft site and two sections at the caudal graft site. In the two control groups [Les-Ctrl (L-DOPA) and Les-Ctrl (Drug-Naive)] the optical density readings on the lesioned side were 7.4 to $10.2 \%$ of the intact side values, and did not differ from each other significantly (Compare Fig 2A, B and C, D, Fig 3B). In the Caudal graft group, the fiber density was significantly increased to $25.7 \pm 3.4 \%$ in the caudal aspect of the head of striatum, while the rostral part remained depleted and was not different from the lesion control animals (Fig. 2G, H, and Fig. 3B). In the Rostral graft group, the transplants provided fiber innervation mainly in the rostral aspect of the head of striatum $(32.2 \pm 3.0 \%$ of the intact side), with a less dense but significant increase also at the more caudal levels (23.8 $\pm 1.3 \%$ of intact side; Fig. 2E, F, and Fig. 3B).

\section{Graft-induced functional recovery}

Graft survival and function was assessed by monitoring amphetamine-induced rotation in the lesioned and grafted animals. In the pre-transplantation test the animals in all groups displayed strong rotational bias towards the side of the lesion $(9.5 \pm 1.0$ to $10.0 \pm 0.7$ turns $/ \mathrm{min}$, Fig. 4$)$. The second amphetamine rotation test was performed at 13 weeks after the grafting, to assess the presence of a surviving functional graft. All but three grafted animals showed prominent reductions in their rotational scores, leading to complete reversal, and even overcompensation (i.e., induction of contralateral turning) in some cases (Fig. 4). The three non-recovered rats [with rotation scores of $10.0,10.0 ; 16.3,24.7$; and 8.5, 15.9 turns/min (pre and post-grafting, respectively)] were excluded from the study, on the assumption of poor graft survival. These animals survived for the remaining part of the in vivo analysis and their brains were processed

for histological examination. Two of the animals displayed very low number of cells $(<200)$ in the striatum, while the third had a graft mis-placed in the piriform cortex. 


\section{Modulation of L-DOPA-induced dyskinesia by intrastriatal VM transplants}

Stable baseline dyskinesia, equivalent to peak-dose dyskinesias seen in PD patients, was achieved by daily L-DOPA injections at a dose of $6 \mathrm{mg} / \mathrm{kg}$ (in combination with $15 \mathrm{mg} / \mathrm{kg}$ benserazide) over 6 weeks. This dose is sufficient to induce behavioral improvement but subthreshold for induction of dyskinesia in non-primed 6-OHDA lesioned rats. However, the same dose of L-DOPA will induce dyskinesia in a significant proportion of these animals upon repeated, daily injections (Winkler et al., 2002). Consistent with previous reports (Cenci et al., 1998, Lee et al., 2000, Lundblad et al., 2002, Winkler et al., 2002) abnormal movements seen in the present animals consisted of dyskinesia involving forelimb and orolingual muscles (predominately hyperkinesia), and dyskinesia in the trunk muscles (essentially dystonia; see movie 1). In addition, some of the animals showed increased locomotion expressed as a contralateral circling movement ("rotation"), in the direction away from the lesioned side (see movie 1). The onset and progression of the severity of dyskinesia varied between rats. Twothirds of the L-DOPA-treated rats had developed dyskinesia after seven days of L-DOPA treatment, and by the end of the $4^{\text {th }}$ week all rats had become dyskinetic and reached a plateau. The dyskinesia were temporally linked to L-DOPA administration, with an onset at 10-15 mins, peak response at 45-90 mins, and a return to baseline by 150-210 mins after each injection (Fig. 5A), analogous to peak-dose dyskinesias seen in PD patients. When evaluated as a group, limb and orolingual dyskinesia constituted $67 \%$ and axial dyskinesia $28 \%$ of the total AIM score. The remaining $5 \%$ was seen as low to moderate grade locomotive dyskinesia (i.e. circling movements away from the lesioned side) and was observed in about $40 \%$ of the treated animals (Fig. 5B). 
AIM tests were carried out at 2, 6, 12, 18 and 24 weeks after transplantation to evaluate the effect of the VM grafts on L-DOPA-induced dyskinesia. As expected, saline-injected lesion control rats [Les-Ctrl (Drug-Naive)] showed no signs of abnormal behaviors, while the animals in the lesion control group [Les-Ctrl (L-DOPA)] that received daily L-DOPA injections maintained a stable dyskinetic response throughout the 24 weeks observation time (Fig. 5C, D). The magnitude of limb and orolingual dyskinesia showed a progressive decline over the postgrafting observation period in the Caudal graft group. This effect became significant at 6 weeks and reached $38.7 \pm 16.5 \%$ of pre-graft value by 24 weeks post grafting. By contrast, no significant effect was seen in the Rostral graft group at any time point (Fig. 5C, D). Axial dyskinesia and locomotive dyskinesia were not improved in any of the grafted groups (Fig. 5D). However, locomotive dyskinesia showed a tendency to be more pronounced in the Caudal graft group, but this difference did not reach significance ( $\mathrm{p}=0.19$; Fig 5D).

Regression analysis using the number of surviving TH-positive neurons in the grafts or THpositive fiber innervation in rostral, caudal, or whole aspect of the head of striatum as independent variables was performed. While none of the individual parameters showed a significant correlation to the total L-DOPA-induced AIM scores $(r<0.1$ and $\mathrm{p}>0.05$ for all comparisons), the severity of both limb and orolingual dyskinesia and axial dyskinesia, as assessed at the 24-week time-point, were inversely correlated to the difference between THpositive fiber reinnervation density in the caudal and rostral aspect of the head of striatum (Fig. 5E, F). The correlation was even more pronounced $(\mathrm{r}=0.81)$ when these two dyskinesia components were combined (Fig. 5G). Only 1 out of 5 rats in the Rostral graft group showed a marked effect on the L-DOPA-induced dyskinesia. This animal (marked with an \# in Fig. 5E-G) had a large graft with fibers reinnervating both areas, thus showing no caudal-rostral difference. 


\section{Graft-induced dyskinesia}

All animals in the VM cell transplanted groups, but none of the animals in the control groups, displayed dyskinetic behaviors after injection of d-amphetamine at 13 weeks post grafting. These abnormal behaviors, referred to as GIDs, remained fully expressed in the subsequent tests performed at 20 and 30 weeks after transplantation. These behaviors were similar in type and pattern to those seen after L-DOPA treatment, including limb and orolingual dyskinesia and axial dyskinesia, and to a lesser extent also circular locomotion expressed as a graft-induced turning away from the grafted side (seen as "overcompensation" in the amphetamine-induced rotation test) (see movie 2). The amphetamine-induced GIDs, however, lasted much longer than the LDOPA-induced AIMs, up to 6 hours after injection (Fig. 6A). The two major components of the GIDs, the limb and orolingual dyskinesia (predominantly hyperkinesia) and the axial dyskinesia (essentially dystonia) were significantly more pronounced in the Caudal graft group than in the Rostral graft group (Fig. 6B). The axial dyskinesia appeared to be a strong component of the amphetamine-induced GIDs, approx. 40\% of total AIM scores (Fig. 6B), as compared with LDOPA-induced dyskinesias (approx. 28\% of total AIM scores; Fig 5B). None of these behaviors were observed in the grafted animals in the absence of drug treatment, either after saline injection or after application of a stressful tail-pinch stimulus. A different type of abnormal behavior, not seen after injection of L-DOPA, which involved the head and neck regions, was observed in all grafted animals (see movie 3). This asymmetric "head-nodding" was present throughout the test period in the grafted rats, but was not observed in any of the non-grafted rats. However, there was no difference in magnitude between the two grafted groups. 
Inspection of the individual grafted animals suggested that the amphetamine-induced GIDs were most pronounced in animals where the graft-derived TH-positive fibers innervated the caudal part of the head of striatum but left the rostral areas relatively depleted (Fig. 7). Consistent with this we found that the magnitude of limb and orolingual dyskinesia and axial dyskinesia was significantly correlated with a relatively higher reinnervation density in the caudal part of the head of striatum (Fig. 6C, D). As was the case for the graft-induced reduction in the L-DOPAinduced dyskinesia (Fig. 5G), the highest correlation $(\mathrm{r}=0.80)$ was obtained when the two dyskinetic components were combined (Fig. 6E). Furthermore, among all grafted animals the limb and orolingual dyskinesia induced by amphetamine and L-DOPA showed a significant inverse correlation $(r=0.67, p=0.02$, not shown). Figure 7 illustrates three representative cases. Case 1 and 2 illustrate two animals from the Caudal graft group that displayed prominent graftinduced limb and orolingual dyskinesia and axial dyskinesia. In these animals the graft-derived reinnervation was confined to parts of the caudal aspect of the head of striatum, and in Case 1, only a circumscribed ventro-lateral part of the caudal part was reinnervated by the graft. These two animals showed also a marked, over 90\%, reduction of L-DOPA-induced limb and orolingual dyskinesia. Case 3 illustrates an animal from the Rostral graft group where the THpositive reinnervation was mostly confined to the central part of the rostral aspect of the head of striatum. This animal displayed little amphetamine-induced GID, and only a marginal reduction in L-DOPA-induced dyskinesia. 


\section{Discussion}

The results show that 6-OHDA lesioned animals, made dyskinetic by prior chronic intermittent L-DOPA treatment, are prone to develop GIDs following transplantation of fetal VM cells when the grafts were activated with amphetamine. Grafts placed in the caudal aspect of the head of striatum, in a region corresponding to the putamen in primates, were effective in reducing already established L-DOPA-induced limb and orolingual dyskinesia (predominately hyperkinesia) by about $60 \%$, but at the same time induce severe dyskinesia when activated with amphetamine. These GIDs were much less pronounced in animals where identical grafts were placed in the rostral aspect of the head of striatum.

\section{Reversal of L-DOPA-induced dyskinesia}

The results highlight the caudal-lateral sector of the head of striatum as a sensitive area for the induction of GIDs, particularly those involving stereotypic forelimb and orolingual movements. As illustrated in Fig. 8, this dyskinesia-prone region shows marked and sustained up-regulation of prodynorphin (PDyn) and $\mathrm{FosB} / \Delta \mathrm{FosB}$-like proteins in rats made dyskinetic by chronic LDOPA treatment (Cenci et al., 1998, Andersson et al., 1999, Winkler et al., 2002, Carlsson et al., 2005). This is also the region where intrastriatal microinjections of amphetamine are particularly efficient in eliciting stereotypic orolingual movements (Kelley et al., 1988, Kuczenski and Segal, 1999, Canales et al., 2000), and it is the area of the lateral striatum that receives direct inputs from the forelimb and orofacial areas of the motor cortex (Ebrahimi et al., 1992, Brown and Sharp, 1995). The ability of the caudal grafts to reverse L-DOPA-induced dyskinesia may be due to partial reinnervation of this dyskinesia-prone area by the grafted DA neurons. This is supported by the time-course of this effect, which became significant at 6 weeks, i.e. at the time 
when the graft-derived TH-positive axons have grown to form a new terminal network, and it was fully developed at 24 weeks when the graft-derived reinnervation of the host striatum is likely to have reached its full extent. The caudal grafts were in most cases located centrally in the striatum, just outside the dyskinesia-prone area, and although the associated terminal network extended into this area it covered in most cases only part of it. In two cases illustrated in Fig 7A,B \& C,D, this partial reinnervation was sufficient to reduce L-DOPA-induced forelimb and orolingual dyskinesia by over $90 \%$.

Previous studies have shown that DA released from the graft-derived terminals can diffuse over some distance and partially normalize DA receptor supersensitivity not only within the area covered by the outgrowing axons, but also in non-reinnervated areas at greater distances from the grafts (Cenci et al., 1992, Chritin et al., 1992, Savasta et al., 1992, Cenci et al., 1993). DA released from a regionally restricted graft-derived terminal network may thus provide a continuous, tonic activation of denervated striatal DA receptors over areas that extend well beyond that reached by the outgrowing fibers. This mechanism may be sufficient to reduce, or in some cases completely reverse, the dyskinesias induced by chronic intermittent L-DOPA delivery. In a previous study, Lee et al., (2000) have shown that as little as 10-20\% reinnervation is sufficient to reduce or completely reverse the excessive up-regulation of preproenkephalin (PPE), PDyn and GAD mRNAs in dyskinetic animals, and that the normalization of these postsynaptic activity markers were highly correlated with the reduction in L-DOPA-induced dyskinesia in the grafted rats.

\section{Induction of amphetamine-induced GIDs}


There is evidence from previous studies that DA receptor supersensitivity is not completely normalized in non-reinnervated or sparsely innervated areas. Stromberg et al., (2000) have shown that spontaneous neuronal firing remains up-regulated and the response to a mixed D1/D2 agonist (apomorphine) remains abnormal in non-reinnervated areas. Similarly, Cenci et al., (1993) have observed that the expression of preprotachykinin (PPT) mRNA, which is reduced in DA-denervated striatal areas, is normalized only in densely reinnervated areas, but not further away from the grafts. These data suggest that the amphetamine-induced GIDs observed here may be caused by excessive release of DA from the graft-derived terminals acting on supersensitive receptors in adjacent non-reinnervated or sparsely reinnervated striatal areas. The limb and orolingual dyskinesia, in particular, may be triggered by DA acting on supersensitive receptors within the dyskinesia-prone caudal-lateral sub-region of the head of striatum, as illustrated in Fig. 8.

Contralateral turning, away from the grafted side, is an abnormal behavior commonly seen in VM transplanted animals in response to an amphetamine challenge. However, the mechanism underlying this response is likely to be quite different from the GIDs involving abnormal limb, orolingual and axial movements. Thus amphetamine-induced contralateral turning, when present, is seen after a single amphetamine injection in non-primed animals, and can appear in the absence of any other signs of dyskinetic behaviors. In the present animals amphetamine-induced contralateral turning was similar in magnitude in both the Rostral and Caudal graft groups, and may thus be elicited from a wider area of the striatum. In a previous study (Cenci et al., 1993) have shown that amphetamine-induced contralateral turning is associated with an excessive induction of fos-immunoreactivity in striatal target neurons within a wider lateral sector of the 
striatum. This response may thus be triggered by excessive graft-derived DA release within a much larger region of the striatum extending far beyond the critical region for induction of GIDs.

Since only L-DOPA treated animals were studied in the present experiment we do not know to what extent L-DOPA priming plays a role in the development of GIDs. The amphetamineinduced GIDs were conspicuously similar to the dyskinesias induced by chronic L-DOPA treatment and may also be triggered from the same striatal sub-region. It seems quite possible, therefore, that once L-DOPA-induced dyskinesia is fully established it may easily be triggered by DA release from grafted DA neurons. Thus, the same cellular mechanisms, possibly involving abnormal activation of striatal cells in a specific sub-region, may be involved in the initiation of these abnormal involuntary movements. The study by Steece-Collier and collaborators has provided some evidence that DA neuron grafts might induce enhanced L-DOPA-induced forelimb hyperkinesia (Steece-Collier et al., 2003). However, in their study, the L-DOPA treatment was initiated already 24 hours after the grafting procedure. As the maturation of VM grafts is extended over several weeks, there is sufficient time for L-DOPA priming to take place before the grafts have provided a terminal fiber network with capacity to take up and store the DA synthesized from L-DOPA.

\section{Clinical implications}

The GIDs in the present animals are different from those seen in grafted PD patients in that they are observed only after activation by amphetamine, while the dyskinesias seen clinically occurred in the OFF-state, i.e. in the absence of any drug challenge. Nevertheless, the present results highlight two major factors of potential importance for the induction of GIDs: the placement of the grafts, and the pattern of reinnervation within the head of the striatum. An 
uneven, patchy reinnervation may create a situation where a sub-threshold, or marginally effective, graft is interacting with postsynaptic targets that remain supersensitive to DA receptor activation. If this model is correct, the GIDs seen in PD patients may be linked to the presence of small grafts that provide a patchy, uneven reinnervation of the putamen. This effect may be particularly pronounced if the grafts are placed in the posterior putamen, i.e. close to the areas that showed localized increases in the $\left[{ }^{18} \mathrm{~F}\right]$-DOPA PET scans in the dyskinetic patients (Ma et al., 2002). The GIDs studied here were correlated with the magnitude of the difference in reinnervation between the caudal and rostral aspect of the head of striatum. This implies that a more widespread, even innervation of larger areas of the striatum would be less prone to induce GIDs. Alternatively, it seems possible that induction of GIDs is primarily determined by the presence or absence of grafts deposits in certain critical sites, rather than the overall magnitude or distribution of the graft-derived reinnervation.

In the rat model of PD, we observed the GIDs only after amphetamine activation, and not in the absence of drug treatment. This suggests that GIDs are induced not only postsynaptically (by supersensitive receptors on striatal target neurons), but that there is an important presynaptic mechanism involved as well, related to excessive or dysregulated DA release from the graftderived terminals. It seems possible that dysregulated DA release could be an important factor also in transplanted PD patients. Data available so far, however do not provide any evidence for overgrowth of the grafted DA neurons, or excessive DA release in patients that develop offphase dyskinesia (Hagell et al., 2002). The GIDs develop slowly over time, and appear to be most pronounced in patients that have received no immunosuppression (Freed et al., 2001) or only mild immonosuppressive treatment (Olanow et al., 2003). In the Olanow et al., (2003) trial one of the troublesome findings was the infiltration of the grafts by activated microglia. It is 
well-known that immune rejection of allografts in the brain is a slow and protracted process and that the grafted DA neurons may survive for a long time but that they might be compromised in function (Duan et al., 1995, Shinoda et al., 1996). Indeed, in the Olanow et al., (2003) study the patients' improvement in unified PD rating scale scores was diminished following withdrawal of the immunosuppression at 6 months, while the survival of the graft did not seem to be affected, as assessed by $\left[{ }^{18} \mathrm{~F}\right]$-DOPA PET. It seems possible that such an ongoing inflammatory/immune process could affect the way the grafted DA neurons release and/or handle DA at the synaptic level, which in turn may constitute a triggering factor for the induction of dyskinesias. Based on the present data we propose that the risk of developing GIDs is highest in patients with small marginally functional grafts that provide an incomplete, patchy reinnervation of the host striatum, particularly when placed in or close to the dyskinesia-prone areas in the putamen. The importance of prior L-DOPA priming and ongoing immune/inflammatory responses need to be investigated further. 


\section{Figure legends}

Figure 1. Experimental design. The experiment was initiated by unilateral complete lesion of the ascending DA pathway in the midbrain (shown at -19 weeks on the time-line). The success of the lesions was evaluated 2 weeks after the 6-OHDA-lesion surgery using amphetamine-induced rotation. The selected animals were then put on daily L-DOPA injection regimen for 6 weeks (induction phase, dark-gray area) to establish a stable dyskinetic response, during which time AIMs were evaluated. A group of well lesioned rats $(n=8)$ were kept as drug-naive lesion controls [Les-Ctrl (Drug-Naive)]. The L-DOPA treated animals displaying moderate to severe AIMs were then balanced into three separate groups, of which two received transplantation of VM cells in the rostral or in the caudal aspect of the head of striatum. A separate, third group, of rats [Les-Ctrl (L-DOPA), $\mathrm{n}=7$ ] were sham-operated and followed as L-DOPA treated lesion controls. To test the effect of the graft on the motor behavior, AIM tests were conducted throughout the experiment between 2 and 24 weeks after the transplantation, as indicated on the timeline. In the dyskinesia maintenance phase (weeks -5 to 29 ; light-gray shaded area), the animals were kept on twice weekly drug treatment. At 13 weeks, an additional amphetamineinduced rotation was conducted to evaluate the functionality of the grafts. Furthermore, at 20 and 30 weeks after transplantation, AIM tests were conducted following activation with $2.5 \mathrm{mg} / \mathrm{kg}$ of d-amphetamine sulfate. The experiment was terminated after the last amphetamine-AIM test by paraformaldehyde perfusion of the animals.

Figure 2. TH-positive fiber re-innervation in the striatum. The grafted TH-positive neurons and their terminal fibers were visualized in the anterior $(\mathrm{E}, \mathrm{G})$, and the posterior striatum $(\mathrm{F}, \mathrm{H}) \mathrm{using}$ immunohistochemistry. The complete lesion paradigm used in this experiment leads to extensive 
depletion of intrinsic nigrostriatal TH-positive fibers, as can be seen by comparison of the striatal sections on the lesioned side $(\mathrm{C}, \mathrm{D})$ and the contralateral intact striatum $(\mathrm{A}, \mathrm{B})$ in the lesion control groups. In animals that received VM graft in the rostral aspect of the part of striatum, a graft core and dense fiber outgrowth were seen in the same sector (E), and less so in the caudal part (F). In the animals that received the VM graft in the caudal aspect of the head of striatum, both transplanted cells and a dense and fiber network was present in the caudal part $(\mathrm{H})$, while no significant fiber outgrowth reached the rostral part (G). Scale bar in $\mathrm{H}$ represents $1 \mathrm{~mm}$ and applies to A-H. cc: corpus callosum, LV: lateral ventricle, STR: striatum.

Figure 3. Quantitative morphological analysis. The number of TH-positive cells (A) and the density of fiber innervation (B) in the striatum were determined by stereological estimation techniques and optical density measurements, respectively. In the transplanted groups single deposits of about 130.000 cells were placed in either the rostral or the caudal aspect of the head of striatum, respectively. The number of TH-positive cells that survived long-term in these groups was similar (2577-3339 cells, representing 19.8-25.7\% survival for the TH expressing cells), and did not differ significantly from each other (A). The optical fiber density (B) was analyzed both at the rostral and caudal aspect of the head of striatum as mean of two levels for each measurement. The two lesion control groups [Les-Ctrl (L-DOPA) and Les-Ctrl (DrugNaive)] did not differ from each other and showed about $90 \%$ reduction as compared with intact striatum. In the grafted groups, significant increases in fiber density were detected in the rostral part in Rostral graft group and in the caudal part in Caudal graft group. However, in the Rostral graft group, there was less prominent but significant fiber outgrowth in the caudal part of the head of striatum as well. * = different from the lesion control groups at rostral striatal level [Oneway ANOVA $\mathrm{F}(2,25)=22.82, \mathrm{p}<0.0001$, followed by student's t-test $] ; \dagger=$ different from the 
lesion control groups at caudal striatal level [One-way ANOVA $\mathrm{F}(2,25)=34.28, \mathrm{p}<0.0001$, followed by student's t-test]

Figure 4. Amphetamine-induced rotation. Amphetamine-induced rotational behavior was conducted at 2 weeks after the 6-OHDA injections, to evaluate the extent of the lesion in the nigrostriatal pathway, and at 13 weeks after transplantation to evaluate the functionality of the grafts. Following the pre-graft testing all animals fulfilling the inclusion criteria was allocated into balanced groups. The two lesion control groups (Les-Ctrl (L-DOPA) and Les-Ctrl (DrugNaive)) showed no improvement in the rotational behaviors, whereas in the two transplanted groups, the animals displayed compensation in their rotational response. $*=$ different from the lesion control groups and their own pre-graft values [two-way repeated measures ANOVA $(\mathrm{F}(7,51)=26.70, \mathrm{p}<0.0001$ followed by Tukey HSD post hoc test $]$.

Figure 5. L-DOPA-induced dyskinesia. The time-course of the L-DOPA-induced AIM scores after a single injection in stably dyskinetic animals prior to grafting is shown in A. The dyskinesias are classified in different sub-categories of behaviors involving to largest extent limb \& orolingual dyskinesia (predominately hyperkinesia) and axial dyskinesia (essentially dystonia), where the first consist of about $67 \%$ and the latter about $28 \%$ of the total AIM scores. In addition increased locomotion in the form of full body rotations is also seen in some (7/18) animals, representing about 5\% of all dyskinesias (B). AIM tests were conduced at various times throughout the study. The time-course of changes in dyskinesias were evaluated from the integrated AIMs scores and the limb and orolingual dyskinesia are expressed here as percent of pre-grafting values (C). Grafts positioned in the caudal aspect of the head of striatum (Filled boxes) were more effective in reversing the limb and orolingual dyskinesia, in comparison to the grafts positioned in the rostral part (Filled circles), (C). While the most pronounced reductions 
were seen in limb and orolingual dyskinesia, the axial dyskinesia were less affected, here shown at the pre and the 24-week post-grafting time-points (D). No significant differences were seen in locomotive dyskinesia in none of the two transplanted groups. However, it is worth noting, that the locomotive dyskinesia in the Caudal graft group showed a tendency to increase, but did not reach significance. Regression analyses showed a significant correlation between the L-DOPAinduced dyskinesias and the difference in TH-positive fiber reinnervation (expressed as \% of intact side values) in the caudal aspect of the head of striatum and the rostral part (E-G). Note that one of the five animals in the Rostral graft group (marked with \#) showed marked reduction in L-DOPA-induced dyskinesia. This animal had a large graft with fibers reinnervating both rostral and caudal areas $*=$ different from the L-DOPA treated lesion controls [Les-Ctrl (LDOPA)], [two-way repeated measures ANOVA $(\mathrm{F}(17,107)=4.20, \mathrm{p}<0.0001$, followed by Tukey HSD post hoc test]; $\uparrow$ different from the pre value, $[\mathrm{p}<0.004$, student t-test $]$

Figure 6. Graft-induced dyskinesia triggered by amphetamine. These amphetamine-induced dyskinetic behaviors were similar to the L-DOPA-induced dyskinetic behaviors and could be classified and scored using the same rating scale. However, these were present for much longer period of time (up to 6 hours, Panel A). Panel B shows the brake-down into three sub-categories [limb \& orolingual dyskinesia (predominantly hyperkinesia), axial dyskinesia (essentially dystonia) and contralateral turning] at 30 weeks after transplantation. All transplanted animals displayed limb and orolingual and axial dyskinesia, however they were more prominent in animals with grafts placed in the caudal aspect of the head of striatum. The frequency of each dyskinesia subcategory within each bar graph in panel B refers to percentage of the total dyskinetic scores. The axial dyskinesia was a strong component of the GIDs and constituted about $40 \%$ of the total dyskinesia score in all grafted animals. Regression analyses showed a 
significant correlation between the GIDs and the difference in TH-positive fiber reinnervation (expressed as \% of intact side values) in the caudal aspect of the head of striatum and the rostral part $(\mathrm{C}-\mathrm{E}){ }^{*}=$ different from Rostral graft group and $\dagger=$ different from Les-Ctrl (L-DOPA) [Limb and orolingual dyskinesia (one-way ANOVA $(F(2,17)=20.41, p<0.0001$ followed by student's t-test); Axial dyskinesia (one-way ANOVA $(\mathrm{F}(2,17)=26.43, \mathrm{p}<0.0001$ followed by student's t-test]

Figure 7. Distribution of graft-derived TH-positive reinnervation in three illustrative cases. Case 1 and 2 show two animals in the Caudal graft group and case 3 one in the Rostral graft group. Individual dyskinesia scores (L-DOPA- and amphetamine-induced GID) are displayed for each case. The localization of the graft and the extent of TH-positive fibers are illustrated at a rostral (A, C, E) and a caudal level (B, D, F). The two caudal graft animals (A, B and C, D) showed a marked (over 90\%) reduction in L-DOPA-induced limb and orolingual dyskinesia (predominately hyperkinesia) and prominent amphetamine-induced GID in the 30 -week postgrafting test. In these two rats the graft-derived reinnervation was confined to the caudal aspect of the head of striatum, and in Case 1 selectively in the ventro-lateral sector, which may serve as a sensitive area for induction of limb and orolingual dyskinesia. The transplant in case 3 by contrast, had minimal effect or either L-DOPA or amphetamine-induced dyskinesia. The reinnervation in this case was mainly confined to rostral part of the head of striatum. Scale bar in panel $\mathrm{F}$ represent $1 \mathrm{~mm}$ and applies to panel A-F). cc: corpus callosum, LV: lateral ventricle, STR: striatum.

Figure 8. Proposed model for induction of AIMs in grafted animals. This drawing represent a coronal view of the forebrain at the level of the striatum overlaid an top of a blurred photo of a TH-stained section showing the graft site and the associated fiber outgrowth in a grafted animal. 
Three important regions in the lateral striatum that has been reported to be involved in activation of the limb and orolingual movements are demonstrated. First, a activation of from the motor cortex has been shown to activate a discrete region in the caudal-lateral striatum (blue striped area, as detrmined by $\left[{ }^{14} \mathrm{C}\right]$ deoxyglucose autoradiography) (Brown and Sharp, 1995). Second, amphetamine has been shown to induce orofacial stereotypy when infused locally in the most ventrolateral aspect of the striatum (black striped area) (Kelley et al., 1988, Canales et al., 2000). Third, these two areas overlap with the area of up-regulation of prodynorphin mRNA message in the caudal-lateral striatum, as seen in dyskinetic rats where abnormal limb and orolingual movements are induced (dyskinesia-prone area; red striped area) (Cenci et al., 1998, Winkler et al., 2002, Carlsson et al., 2005). It is proposed that the ability of the caudally placed grafts to normalize L-DOPA-induced limb and orolingual dyskinesia (predominately hyperkinesia) and to induce GIDs after amphetamine activation, is caused by partial reinnervation, and diffusion of released DA into this dyskinesia-prone area in the ventral-lateral striatum, as indicated by the red arrows. Scale bar represent $2 \mathrm{~mm}$. cc: corpus callosum, CTX: Cortex, LV: lateral ventricle, AC: anterior commissure. 


\section{Movie legend:}

The abnormal involuntary movements displayed in these animals were generated over a 6-week daily administration protocol of $6 \mathrm{mg} / \mathrm{kg}$ L-DOPA (+ $15 \mathrm{mg} / \mathrm{kg}$ Benserazide-HCl, the peripheral DOPA-decarboxylase inhibitor). After this induction period, the dyskinesias were maintained by twice-weekly L-DOPA injections over 34 weeks (weeks -5 to 29). The three movies were videotaped at various times during the course of the experiment. While movie 1 are recorded at peak-dose response interval after a single dose of L-DOPA, movie 2 and 3 were filmed from grafted animals during the AIM testing after activation of the grafts with $2.5 \mathrm{mg} / \mathrm{kg} \mathrm{d}$ amphetamine, at the 20-week time-point.

Movie 1: This part contain four segments; 1: Moderate to severe hyperkinetic movements in the left forepaw, i.e. the side of the lesion; 2: Purposeless chewing and tongue protrusion. Note that the hyperkinetic movements are towards the left side, which is better illustrated in the slow motion sequence in the end; 3: Dystonic twisting of the trunk towards left side. This animal also exhibits limb and orolingual hyperkinetic movements on the same side; 4: Severe abnormal locomotive behavior away from the lesion side.

Movie 2. This part contain four segments 1: Hyperkinetic limb movements induced by the graft. The animal also displays hyperkinetic head movements (referred in the text as "asymmetric head nodding"); 2: Orolingual GIDs. The grafted animal displays purposeless chewing and tongue protrusions after activation with amphetamine, similar to what can be seen in L-DOPA treated animals. Note also the head nodding; 3: Axial GID seen as severe dystonic twisting of the trunk, 
which in this case results in loss of balance; 4: Low-grade contralateral turning following the amphetamine challenge. In addition, the animal displays pronounced axial and limb GIDs.

Movie 3: The animal in this movie display excessive head and neck movements that better illustrate the head nodding behavior (also seen in movie 2, segments 1 and 2).

\section{Acknowledgements}

We thank Ulla Jarl, Anneli Josefsson and Bengt Mattsson for their expert technical support. This study was supported by grants from the Swedish Research Council (K2003-33SX-14552-01A, K2003-33P-14788-01A, K2005-33IT-15332-01A and 2005-33X-14552-03A to DK, K200333X-13480-0A to MAC, and 04X-3874 to AB), and the HiLF program of the Hannover medical School (to CW). 


\section{References:}

Andersson, M., Hilbertson, A. and Cenci, M. A., 1999. Striatal fosB expression is causally linked with 1-DOPA-induced abnormal involuntary movements and the associated upregulation of striatal prodynorphin mRNA in a rat model of Parkinson's disease. Neurobiol Dis. 6, 461-474.

Brown, L. L. and Sharp, F. R., 1995. Metabolic mapping of rat striatum: somatotopic organization of sensorimotor activity. Brain Res. 686, 207-222.

Brundin, P., Pogarell, O., Hagell, P., Piccini, P., Widner, H., Schrag, A., Kupsch, A., Crabb, L., Odin, P., Gustavii, B., Bjorklund, A., Brooks, D. J., Marsden, C. D., Oertel, W. H., Quinn, N. P., Rehncrona, S. and Lindvall, O., 2000. Bilateral caudate and putamen grafts of embryonic mesencephalic tissue treated with lazaroids in Parkinson's disease. Brain. 123 ( Pt 7), 1380-1390. Canales, J. J., Gilmour, G. and Iversen, S. D., 2000. The role of nigral and thalamic output pathways in the expression of oral stereotypies induced by amphetamine injections into the striatum. Brain Res. 856, 176-183.

Carlsson, T., Winkler, C., Burger, C., Muzyczka, N., Mandel, R. J., Cenci, A., Bjorklund, A. and Kirik, D., 2005. Reversal of dyskinesias in an animal model of Parkinson's disease by continuous L-DOPA delivery using rAAV vectors. Brain. 128, 559-569.

Cenci, M. A., Campbell, K. and Bjorklund, A., 1993. Neuropeptide messenger RNA expression in the 6-hydroxydopamine-lesioned rat striatum reinnervated by fetal dopaminergic transplants: differential effects of the grafts on preproenkephalin, preprotachykinin and prodynorphin messenger RNA levels. Neuroscience. 57, 275-296.

Cenci, M. A., Kalen, P., Mandel, R. J., Wictorin, K. and Bjorklund, A., 1992. Dopaminergic transplants normalize amphetamine- and apomorphine-induced Fos expression in the 6hydroxydopamine-lesioned striatum. Neuroscience. 46, 943-957. 
Cenci, M. A., Lee, C. S. and Bjorklund, A., 1998. L-DOPA-induced dyskinesia in the rat is associated with striatal overexpression of prodynorphin- and glutamic acid decarboxylase mRNA. Eur J Neurosci. 10, 2694-2706.

Chritin, M., Savasta, M., Mennicken, F., Bal, A., Abrous, D. N., Le Moal, M., Feuerstein, C. and Herman, J. P., 1992. Intrastriatal Dopamine-rich Implants Reverse the Increase of Dopamine D2 Receptor mRNA Levels Caused by Lesion of the Nigrostriatal Pathway: A Quantitative In Situ Hybridization Study. Eur J Neurosci. 4, 663-672.

Defer, G. L., Geny, C., Ricolfi, F., Fenelon, G., Monfort, J. C., Remy, P., Villafane, G., Jeny, R., Samson, Y., Keravel, Y., Gaston, A., Degos, J. D., Peschanski, M., Cesaro, P. and Nguyen, J. P., 1996. Long-term outcome of unilaterally transplanted parkinsonian patients. I. Clinical approach. Brain. 119 ( Pt 1), 41-50.

Duan, W. M., Widner, H., Frodl, E. M. and Brundin, P., 1995. Immune reactions following systemic immunization prior or subsequent to intrastriatal transplantation of allogeneic mesencephalic tissue in adult rats. Neuroscience. 64, 629-641.

Ebrahimi, A., Pochet, R. and Roger, M., 1992. Topographical organization of the projections from physiologically identified areas of the motor cortex to the striatum in the rat. Neurosci Res. $14,39-60$.

Fahn, S., 2000. The spectrum of levodopa-induced dyskinesias. Ann Neurol. 47, S2-9; discussion S9-11.

Freed, C. R., Breeze, R. E., Fahn, S. and Eidelberg, D., 2004. Preoperative response to levodopa is the best predictor of transplant outcome. Ann Neurol. 55, 896; author reply 896-897. 
Freed, C. R., Greene, P. E., Breeze, R. E., Tsai, W. Y., DuMouchel, W., Kao, R., Dillon, S., Winfield, H., Culver, S., Trojanowski, J. Q., Eidelberg, D. and Fahn, S., 2001. Transplantation of embryonic dopamine neurons for severe Parkinson's disease. N Engl J Med. 344, 710-719.

Georgievska, B., Carlsson, T., Lacar, B., Winkler, C. and Kirik, D., 2004. Dissociation between short-term increased graft survival and long-term functional improvements in Parkinsonian rats overexpressing glial cell line-derived neurotrophic factor. Eur J Neurosci. 20, 3121-3130.

Greene, P. E., Fahn, S., Tsai, W. Y., Breeze, R. E., Eidelberg, D., Winfield, H., Dillon, S., Kao, R., Winfield, L. and Freed, C. R., 1999. Severe Spontanous Dyskinesias: A Disabling Complication of Embryonic Dopaminergic Tissue Implants in a Subset of Transplanted Patients With Advanced Parkinson's Disease. Movement Disorders. 14, 904.

Hagell, P., Piccini, P., Bjorklund, A., Brundin, P., Rehncrona, S., Widner, H., Crabb, L., Pavese, N., Oertel, W. H., Quinn, N., Brooks, D. J. and Lindvall, O., 2002. Dyskinesias following neural transplantation in Parkinson's disease. Nat Neurosci. 5, 627-628.

Hagell, P., Schrag, A., Piccini, P., Jahanshahi, M., Brown, R., Rehncrona, S., Widner, H., Brundin, P., Rothwell, J. C., Odin, P., Wenning, G. K., Morrish, P., Gustavii, B., Bjorklund, A., Brooks, D. J., Marsden, C. D., Quinn, N. P. and Lindvall, O., 1999. Sequential bilateral transplantation in Parkinson's disease: effects of the second graft. Brain. 122 ( Pt 6), 1121-1132. Hauser, R. A., Freeman, T. B., Snow, B. J., Nauert, M., Gauger, L., Kordower, J. H. and Olanow, C. W., 1999. Long-term evaluation of bilateral fetal nigral transplantation in Parkinson disease. Arch Neurol. 56, 179-187.

Jenner, P., 2000. Factors influencing the onset and persistence of dyskinesia in MPTP-treated primates. Ann Neurol. 47, S90-99; discussion S99-104. 
Kelley, A. E., Lang, C. G. and Gauthier, A. M., 1988. Induction of oral stereotypy following amphetamine microinjection into a discrete subregion of the striatum. Psychopharmacology (Berl). 95, 556-559.

Kirik, D., Winkler, C. and Bjorklund, A., 2001. Growth and functional efficacy of intrastriatal nigral transplants depend on the extent of nigrostriatal degeneration. J Neurosci. 21, 2889-2896. Kuczenski, R. and Segal, D. S., 1999. Sensitization of amphetamine-induced stereotyped behaviors during the acute response. J Pharmacol Exp Ther. 288, 699-709.

Lee, C. S., Cenci, M. A., Schulzer, M. and Bjorklund, A., 2000. Embryonic ventral mesencephalic grafts improve levodopa-induced dyskinesia in a rat model of Parkinson's disease. Brain. 123 ( Pt 7), 1365-1379.

Lindvall, O. and Bjorklund, A., 2004. Cell Replacement Therapy: Helping the Brain to Repair Itself. Neurorx. 1, 379-381.

Lundblad, M., Andersson, M., Winkler, C., Kirik, D., Wierup, N. and Cenci, M. A., 2002. Pharmacological validation of behavioural measures of akinesia and dyskinesia in a rat model of Parkinson's disease. Eur J Neurosci. 15, 120-132.

Ma, Y., Feigin, A., Dhawan, V., Fukuda, M., Shi, Q., Greene, P., Breeze, R., Fahn, S., Freed, C. and Eidelberg, D., 2002. Dyskinesia after fetal cell transplantation for parkinsonism: a PET study. Ann Neurol. 52, 628-634.

Marsden, C. D., Parkes, J. D. and Quinn, N., 1982. Fluctuations of disability in Parkinson's disease - clinical aspects. In: disorders, M. (Ed.), Marsden C. D. and Fahn S., London: Butterworth, pp. 96-122. 
Nikkhah, G., Olsson, M., Eberhard, J., Bentlage, C., Cunningham, M. G. and Bjorklund, A., 1994. A microtransplantation approach for cell suspension grafting in the rat Parkinson model: a detailed account of the methodology. Neuroscience. 63, 57-72.

Nutt, J. G., Obeso, J. A. and Stocchi, F., 2000. Continuous dopamine-receptor stimulation in advanced Parkinson's disease. Trends Neurosci. 23, S109-115.

Olanow, C. W., Goetz, C. G., Kordower, J. H., Stoessl, A. J., Sossi, V., Brin, M. F., Shannon, K. M., Nauert, G. M., Perl, D. P., Godbold, J. and Freeman, T. B., 2003. A double-blind controlled trial of bilateral fetal nigral transplantation in Parkinson's disease. Ann Neurol. 54, 403-414. Paxinos, G. and Watson, C., 1998. The rat brain in stereotaxic coordinates. Academic Press, San Diego.

Quinn, N. P., 1998. Classification of fluctuations in patients with Parkinson's disease. Neurology. 51, S25-29.

Savasta, M., Mennicken, F., Chritin, M., Abrous, D. N., Feuerstein, C., Le Moal, M. and Herman, J. P., 1992. Intrastriatal dopamine-rich implants reverse the changes in dopamine D2 receptor densities caused by 6-hydroxydopamine lesion of the nigrostriatal pathway in rats: an autoradiographic study. Neuroscience. 46, 729-738.

Shinoda, M., Hudson, J. L., Stromberg, I., Hoffer, B. J., Moorhead, J. W. and Olson, L., 1996. Microglial cell responses to fetal ventral mesencephalic tissue grafting and to active and adoptive immunizations. Exp Neurol. 141, 173-180.

Steece-Collier, K., Collier, T. J., Danielson, P. D., Kurlan, R., Yurek, D. M. and Sladek, J. R., Jr., 2003. Embryonic mesencephalic grafts increase levodopa-induced forelimb hyperkinesia in parkinsonian rats. Mov Disord. 18, 1442-1454. 
Stromberg, I., Kehr, J., Andbjer, B. and Fuxe, K., 2000. Fetal ventral mesencephalic grafts functionally reduce the dopamine D2 receptor supersensitivity in partially dopamine reinnervated host striatum. Exp Neurol. 164, 154-165.

West, M. J., 1999. Stereological methods for estimating the total number of neurons and synapses: issues of precision and bias. Trends Neurosci. 22, 51-61.

Winkler, C., Bentlage, C., Nikkhah, G., Samii, M. and Bjorklund, A., 1999. Intranigral transplants of GABA-rich striatal tissue induce behavioral recovery in the rat Parkinson model and promote the effects obtained by intrastriatal dopaminergic transplants. Exp Neurol. 155, 165186.

Winkler, C., Kirik, D. and Bjorklund, A., 2005. Cell transplantation in Parkinson's disease: how can we make it work? Trends Neurosci. 28, 86-92.

Winkler, C., Kirik, D., Bjorklund, A. and Cenci, M. A., 2002. L-DOPA-induced dyskinesia in the intrastriatal 6-hydroxydopamine model of parkinson's disease: relation to motor and cellular parameters of nigrostriatal function. Neurobiol Dis. 10, 165-186. 
Figure 1

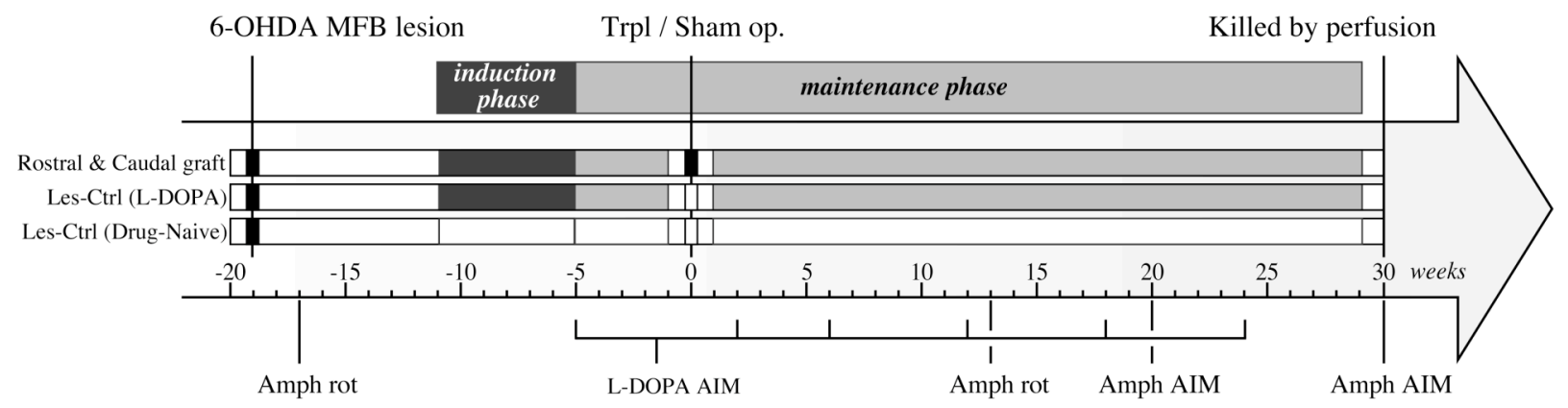


Figure 2

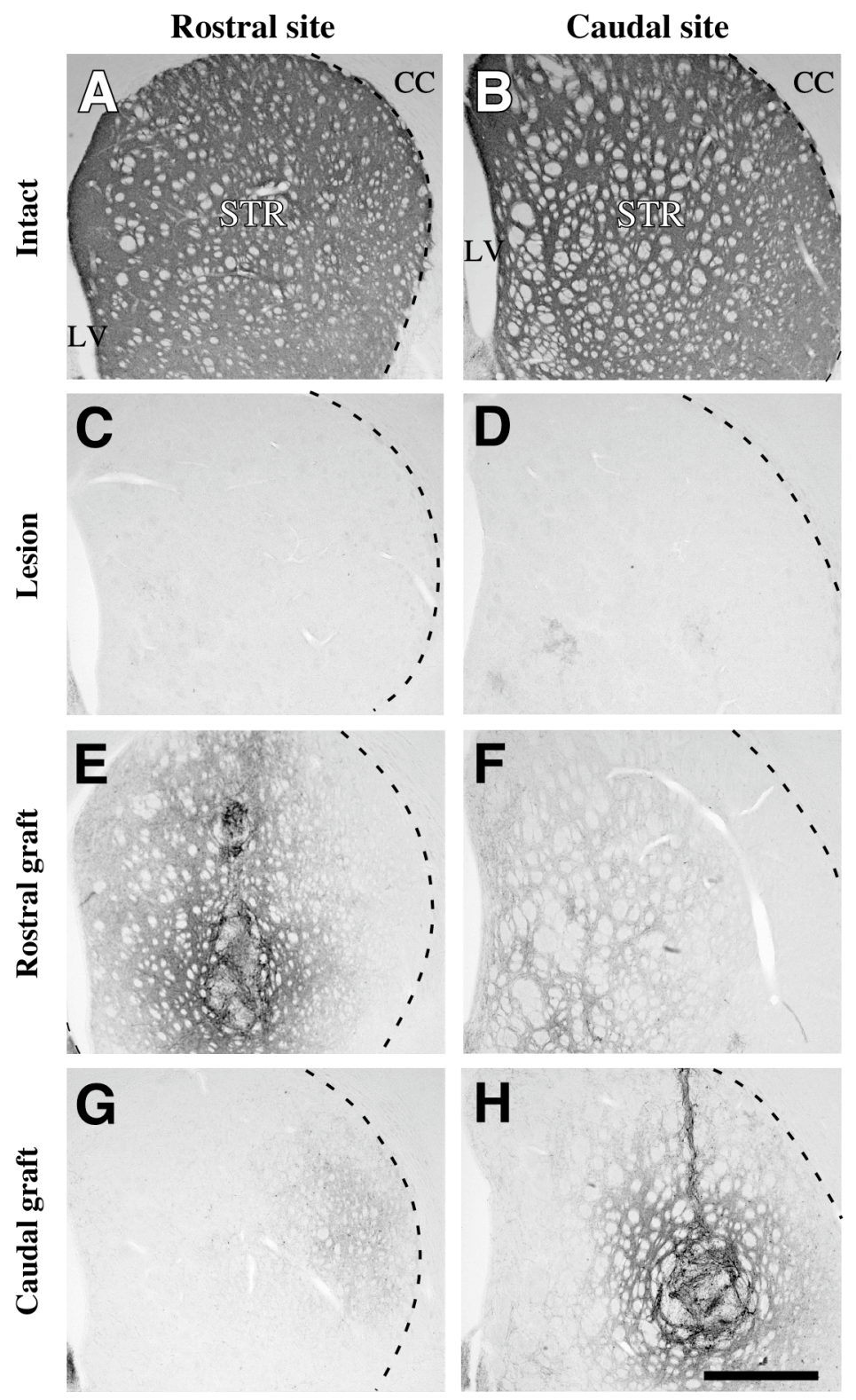


Figure 3

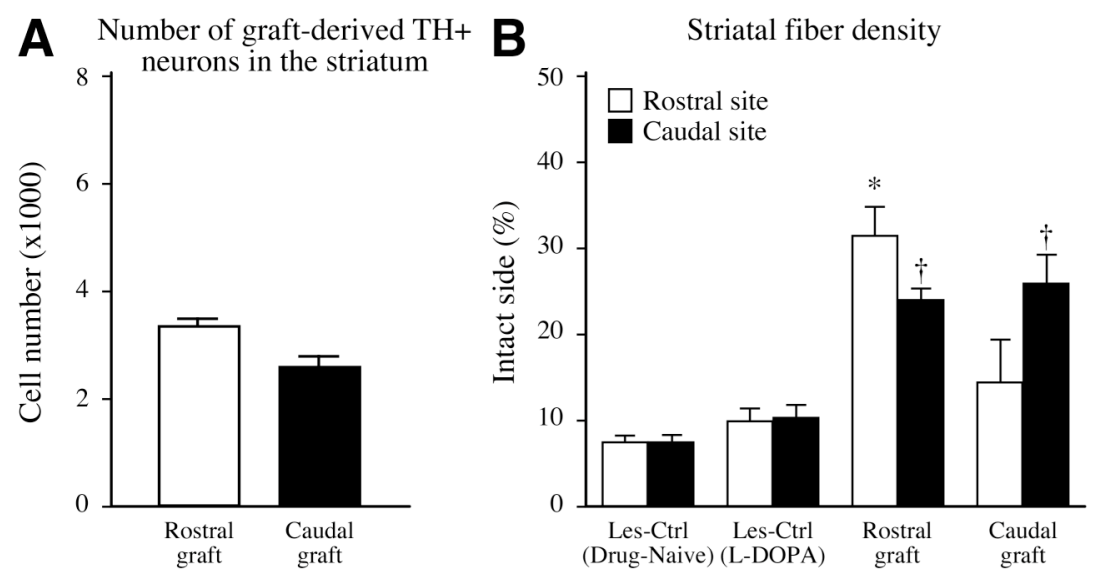


Figure 4

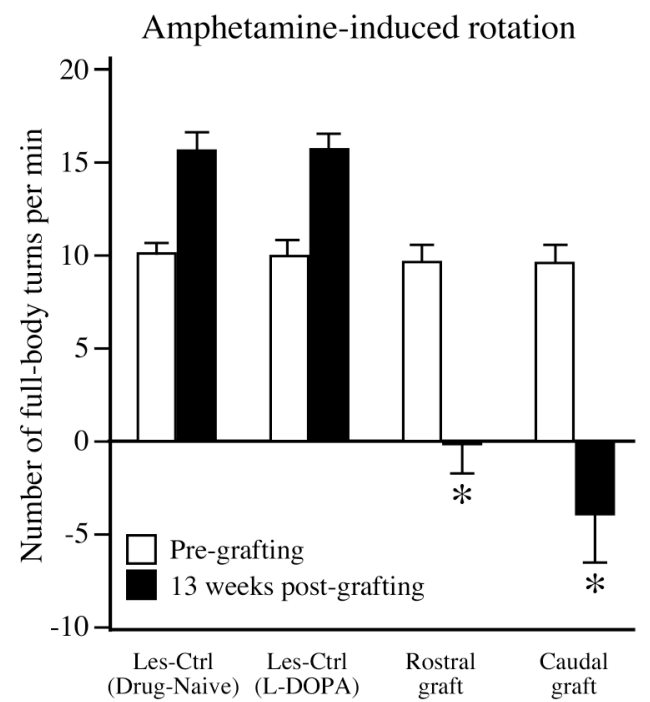


Figure 5
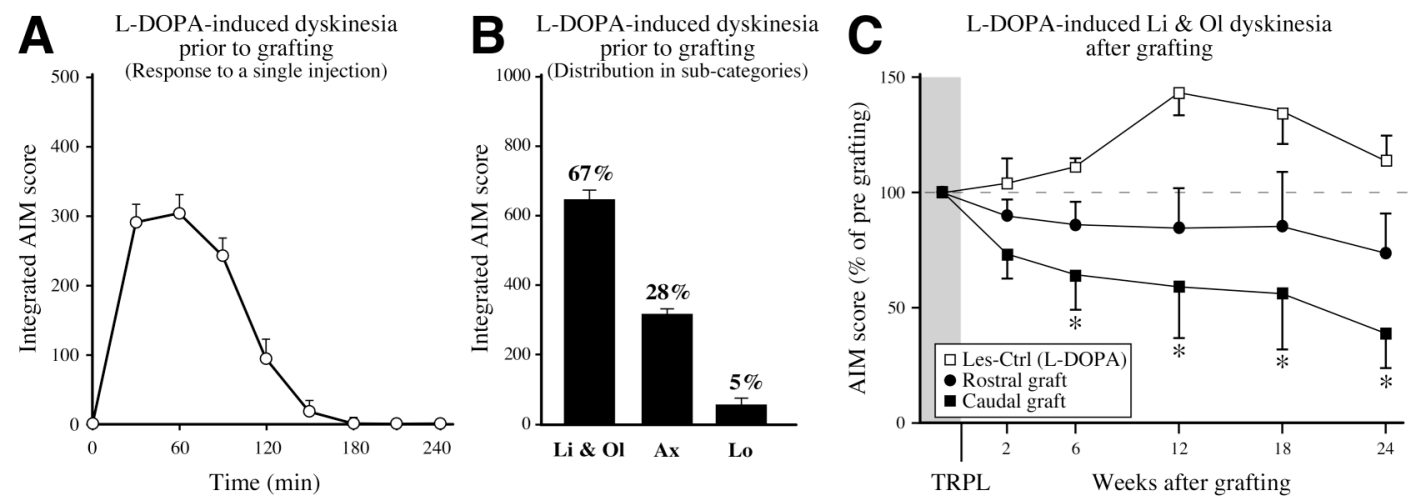

D

L-DOPA-induced dyskinesia pre and at 24 weeks post grafting

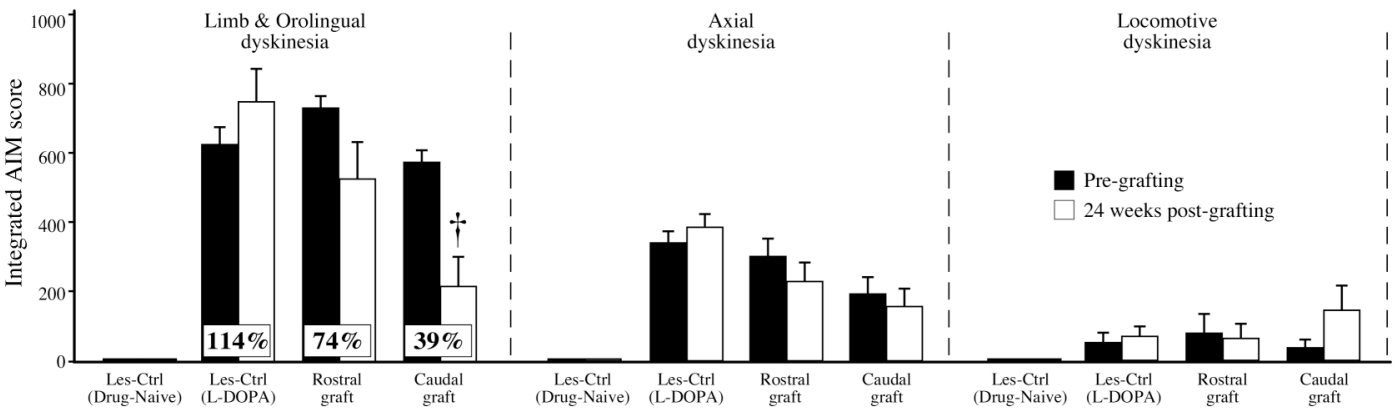

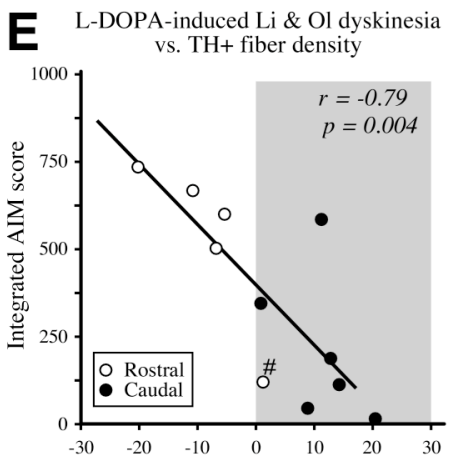

difference in fiber density (caudal - rostral)
E L-DOPA-induced Ax dyskinesia

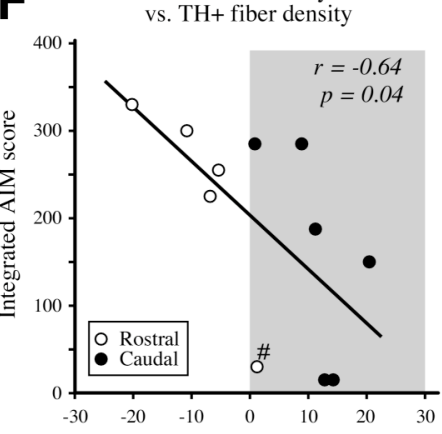

difference in fiber density (caudal - rostral)
G $\begin{gathered}\text { L-DOPA-induced } \mathrm{Li}, \mathrm{Ol} \& \text { Ax dyskinesia } \\ \text { vs. TH+ fiber density }\end{gathered}$

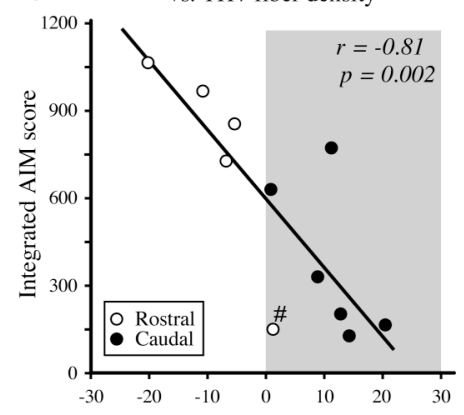

difference in fiber density (caudal - rostral) 
Figure 6

A Graft-induced dyskinesia at 20 wks

B Graft-induced dyskinesia and turning at 30 weeks
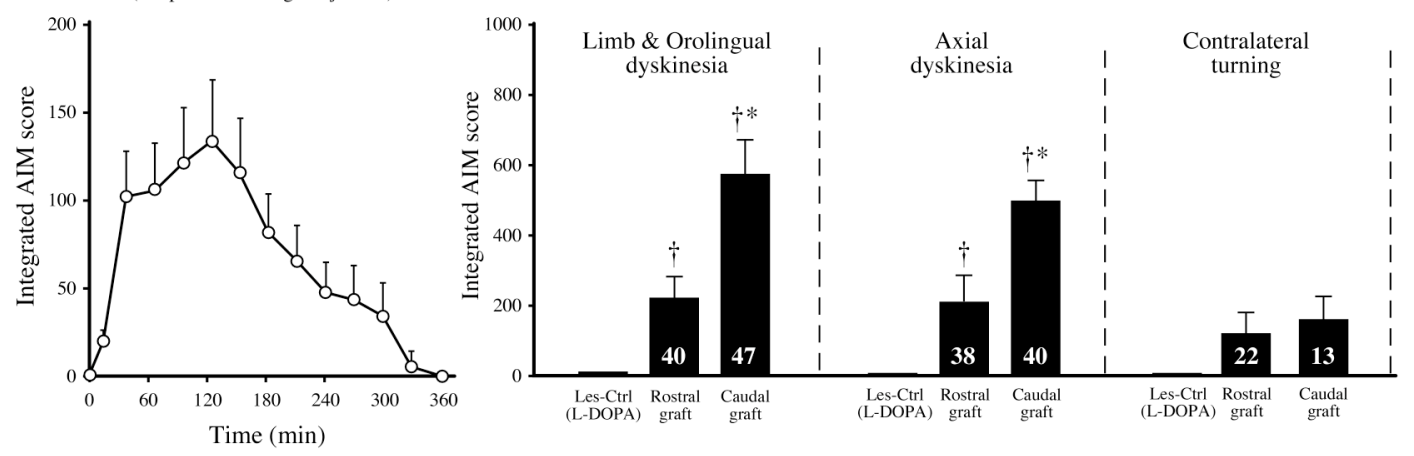

C Graft-induced $\mathrm{Li} \&$ Ol dyskinesia

D Graft-induced Ax dyskinesia
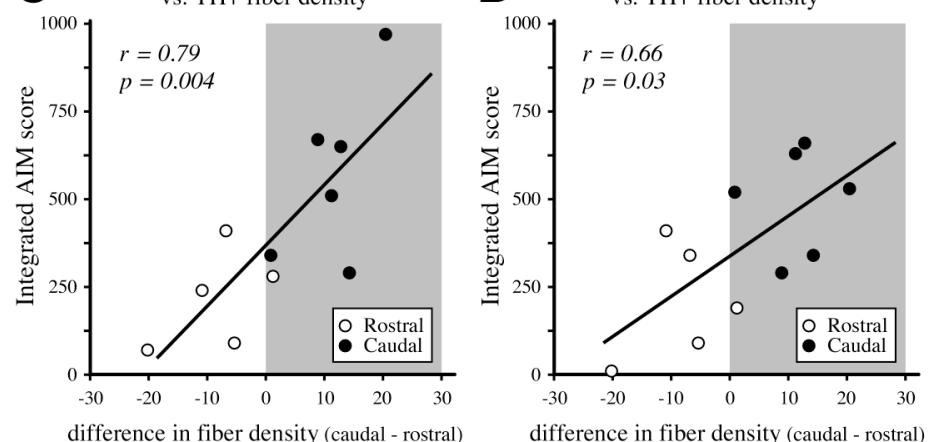

E Graft-induced $\mathrm{Li}, \mathrm{Ol} \&$ Ax dyskinesia

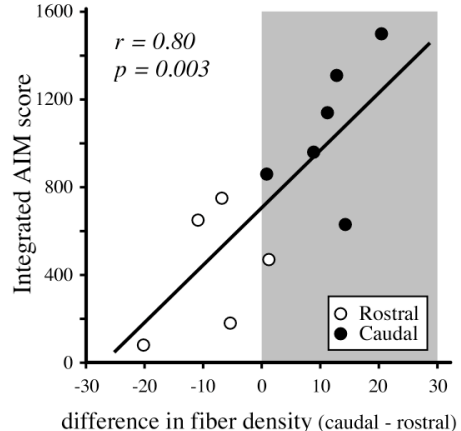

difference in fiber density (caudal - rostral)

difference in fiber density (caudal - rostral)

difference in fiber density (caudal - rostral) 
Figure 7

\begin{tabular}{l|cccccc} 
& \multicolumn{2}{c}{$\begin{array}{c}\text { CASE 1 } \\
\text { Caudal graft }\end{array}$} & \multicolumn{2}{c}{$\begin{array}{c}\text { CASE 2 } \\
\text { Caudal graft }\end{array}$} & \multicolumn{2}{c}{$\begin{array}{c}\text { CASE 3 } \\
\text { Rostral graft }\end{array}$} \\
\cline { 2 - 7 } & Pre & Post & Pre & Post & Pre & Post \\
\hline Amph-induced rotation & 11.0 & 2.3 & 6.1 & -0.1 & 6.7 & 0.5 \\
L-DOPA: Li \& Ol & 570 & 45 & 654 & 15 & 714 & 600 \\
L-DOPA: Ax & 114 & 285 & 270 & 150 & 345 & 255 \\
GID: Li \& Ol & - & 670 & - & 970 & - & 90 \\
GID: Ax & - & 290 & - & 530 & - & 90
\end{tabular}

Caudal site
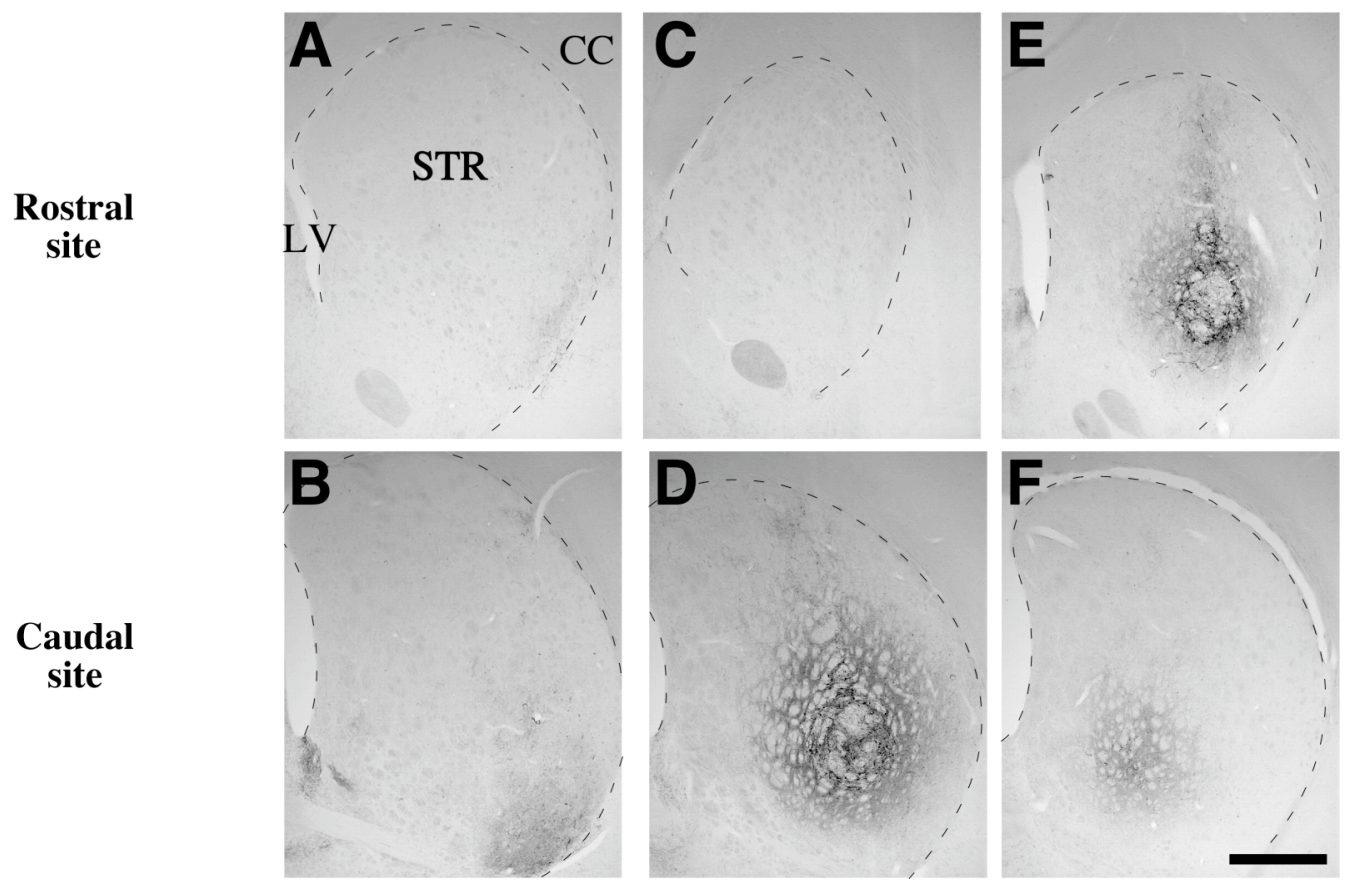
Figure 8

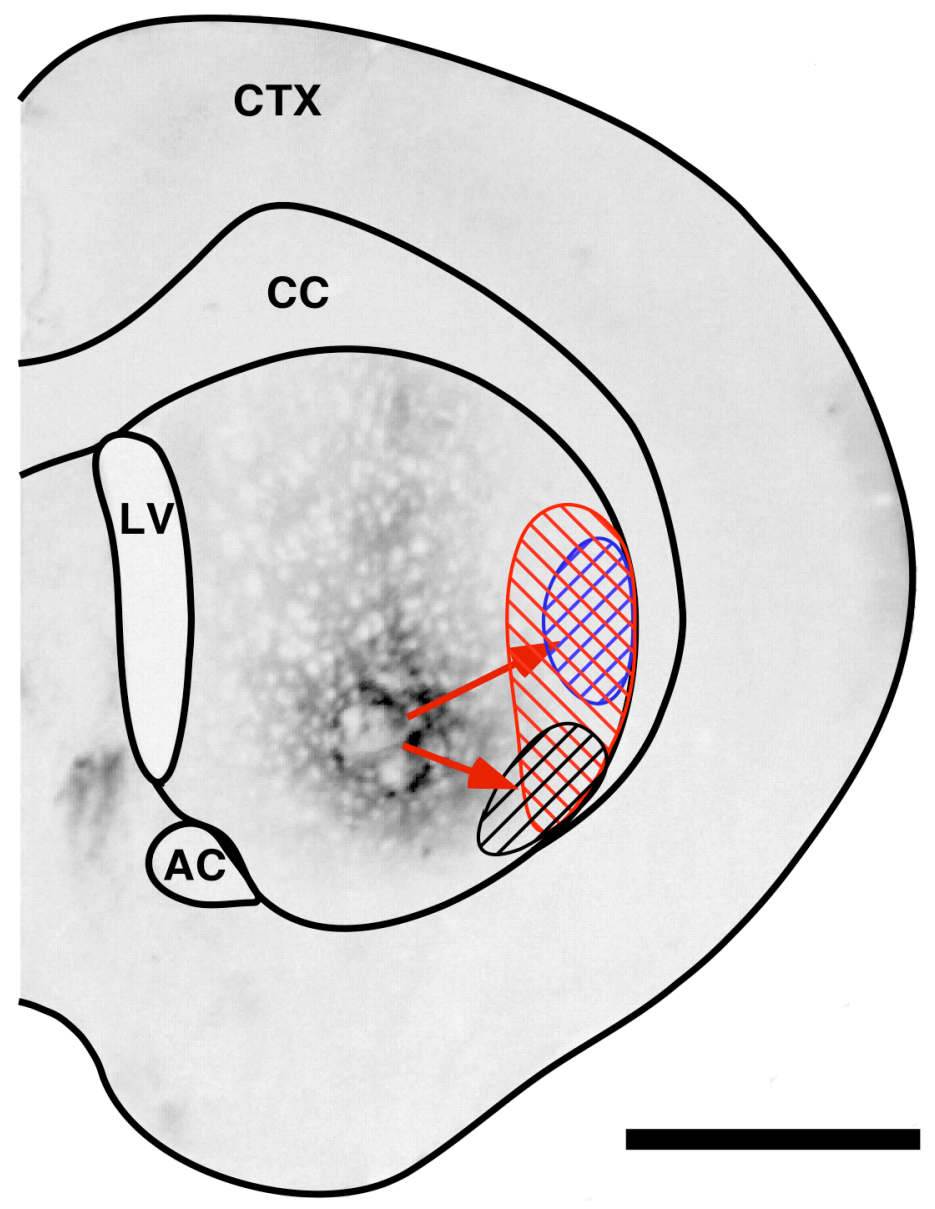

